\title{
Influencia de las variables peso, gap y material en el comportamiento de la placa DCP en fracturas de fémur
}

\author{
MARIANO SAURA SÁNCHEZ, ANICETO VALVERDE MARTÍNEZ, FRANCISCO SAURA SÁNCHEZ, ELADIO \\ SAURA SÁNCHEZ. \\ Universidad Politécnica de Cartagena
}

\section{Introducción y objetivos}

La placa de compresión dinámica $\mathrm{DCP}$ es un sistema de fijación interna indicado para determinado tipo de osteosínesis de fracturas diafisiarias de fémur [1-3]. Esta placa utiliza el principio de compresión dinámica, que consiste en utilizar la geometría específica de los taladros distribuidos sobre la placa para conseguir aproximar los extremos de la fractura hasta ponerlos en contacto e incluso aplicar una cierta compresión entre ellos por el efecto de descentrado de los tornillos.

Estudios realizados por los autores de este trabajo sobre el estado tensional al que se encuentra sometido esta placa, a medida que se incrementa la carga aplicada sobre la articulación coxofemoral, revelan que las tensiones máximas alcanzadas para apoyos de hasta $100 \mathrm{~kg}$ están muy por debajo del límite elástico del material cuando existe contacto entre los extremos de la fractura a nivel de la segunda cortical [4]. Sin embargo, en determinadas circunstancias no es posible conseguir contacto entre los extremos de la fractura. Se llama gap a la distancia entre los extremos del fémur distal a uno y otro lados del foco de fractura. En estos casos la placa de compresión dinámica DCP actúa como puente entre dichos extremos, función que da nombre al efecto que produce: placa puente o también de neutralización.

Cuando la placa DCP actúa en este sentido, sus secciones correspondientes a la altura del foco de fractura deben soportar la transmisión de cargas entre los extremos del fémur por la discontinuidad que genera el gap.

\section{Correspondencia:}

Campus Universitario Muralla del Mar.C/ Doctor

Fleming, s/n.30202 Cartagena

msaura.sanchez@upct.es
A pesar de que se sabe que esta es la condición de trabajo más desfavorable para la placa, y que es en alguna sección de esa zona donde se produce su fracaso, aún hoy día no se ha estudiado detalladamente el estado tensional en esa zona en función del gap de la fractura, del peso del paciente y de las propiedades mecánicas del material en que está fabricado el implante, parámetros que el cirujano puede conocer con exactitud y podría utilizar para determinar si una determinada placa fracasará o no antes de la consolidación de la fractura.

El objetivo de este estudio es, por tanto, analizar mediante el método de elementos finitos, el estado tensional de la placa DCP bajo distintas condiciones de carga (apoyo progresivo), distintos gaps en la fractura y distintos materiales de fabricación.

\section{Método}

En el modelo de elementos finitos se han considerado los siguientes sólidos: placa de osteosíntesis, tornillos de fijación y fémur.

\section{Consideraciones sobre el modelo}

La placa objeto de estudio ha sido la DCP fabricada en acero inoxidable 316LVM Condición Médium/Hard [5] y en titanio Ti5Al7Nb [5,6] compuesta por 14 taladros. Su geometría, tanto externa como la de sus taladros ha sido obtenida de forma precisa mediante una máquina de medición por coordenadas modelo DEA SWIFT A 001.

Los métodos utilizados para la modelización y mallado de la placa, así como de los tornillos de fijación y fémur y las distintas consideraciones e hipótesis de trabajo asumidas para la generación del conjunto han sido expuestos por los autores en un trabajo anterior [4]. Los modelos de placa, hueso y tornillos utilizan un elemento tridimensional 
isoparamétrico de 8 nodos [4]. En la mayoría de los casos, se ha conseguido un mallado mapeado de los distintos sólidos con tamaño de los elementos adaptados a las zonas de mayor interés o de gradiente tensional.

Se han considerado cuatro tornillos de $4.5 \mathrm{~mm}$ de diámetro de caña en los correspondientes taladros proximales y otros cuatro en los distales. El material en que están fabricados los tornillos de fijación es el mismo que el de la placa

El par de apriete que el cirujano aplica sobre el tornillo para fijar la placa al hueso es de $3 \mathrm{Nm}$ [7]

El modelo del fémur es anatómico en su tercio proximal y cilíndrico en su diáfisis. Solo se han considerado propiedades de hueso cortical en todo el fémur.

La zona diafisiaria que estará en contacto con la parte central de la placa se ha dividido en rodajas de $1 \mathrm{~mm}$ de espesor para posibilitar el estudio de fracturas con distintas geometrías (desde $1 \mathrm{~mm}$ hasta $10 \mathrm{~mm}$ ). El resto de la diáfisis del fémur se ha generado en rodajas de aproximadamente $1 \mathrm{~cm}$ para poder estudiar gaps mayores.

El modelo de la placa está compuesto por 31.620 elementos de 8 nodos; el hueso cortical por 42.287 y los tornillos de fijación por 216 elementos de 8 nodos cada uno.

Para modelizar el comportamiento elasto-plástico de un metal mediante MEF se opta por la representación tensión-deformación bilineal en la que se supone linealidad de los tramos elástico y plástico. Una expresión capaz de representar la zona plástica es la ley exponencial desarrollada por LudwikHollomon:

$$
\sigma=K \varepsilon^{n}
$$

El criterio adoptado para determinar si el material sobrepasa la tensión de fluencia es el de Von Mises, ampliamente aceptado para ensayo de metales.

Por último optamos por el endurecimiento isotropico como regla de endurecimiento, que representa el cambio en el límite de fluencia con las progresivas deformaciones plásticas.

\section{Tipo de análisis y estado de cargas}

Se ha realizado un estudio estático tridimensional, considerando comportamiento elastoplástico no lineal, isótropo y homogéneo de todos los materiales que intervienen en el modelo. En este estudio no existe contacto entre los extremos de la fractura en la segunda cortical, pero se considera que éste puede aparecer a medida que la placa se deforme plásticamente. Por ello se han generado 416 elementos de contacto entre los extremos de la fractura tipo superficie-superficie.

La hipótesis de carga corresponde a un apoyo monopodal parcial estático equivalente a la fase 16 el ciclo de la marcha humana [8].

Se utiliza el modelo de E. Genda [9] para calcular las componentes de reacción sobre la articulación [4].

El extremo distal del fémur se ha considerado empotrado [10]

En este estudio se aplican cargas de $100 \mathrm{~N}$ a $300 \mathrm{~N}$ con intervalos de $100 \mathrm{~N}$. Cada uno de los tres estados de carga se aplica a diez hipótesis de GAP de $1 \mathrm{~mm}$ a $10 \mathrm{~mm}$ con incrementos de $1 \mathrm{~mm}$ para cada material.

\section{Resultados}

Se han analizado tres estados de carga para cada uno de los diez GAPs simulados y todo ello para cada uno de los dos materiales estudiados, luego en total se han resuelto sesenta hipótesis de estudio cuyos resultados presentamos en este apartado. Se ha estudiado la distribución de tensiones equivalentes de Von Mises para todas las hipótesis.

Para el acero se observa que los puntos de máxima tensión aparecen: a) Para la cara anterior, en la zona de los taladros próxima al foco de la fractura; que en algunos casos contienen la tensión máxima. B) Para la cara posterior, en la zona del GAP y más acusadamente en las zonas de fin de contacto del hueso. En la figura 1 se muestra el caso de una fractura con Gap de $8 \mathrm{~mm}$, carga de 300 Newton y material acero y titanio. Este caso ilustra la situación de las zonas anteriormente mencionadas, aunque los valores de tensión dependen del GAP, la carga aplicada y el material, como se muestra en la gráfica de la figura 2.

Para el titanio se observa que existe una única zona de máxima tensión situada en el contacto de la placa con la fractura para todos los estados de carga. Solo aparecen zonas de tensiones elevadas en los bordes de los taladros a partir de la carga de 300 Newton.

Una visión completa de los resultados calculados se puede obtener mediante la gráfica de la figura 2, que muestra la tensión máxima en la pieza en función del GAP para distintos estados de carga y materiales

Se puede observar un descenso de la tensión máxima cuando se incrementa el GAP de 1 a 2 
$\mathrm{mm}$, permaneciendo constante con sucesivos incrementos del GAP hasta llegar a GAP $7 \mathrm{~mm}$, donde se produce un incremento de la tensión considerable. A partir de GAP 8, la tensión vuelve a estabilizarse hasta el último GAP de $10 \mathrm{~mm}$ calculado.

Estas valoraciones coinciden tanto para el caso del acero como para el titanio, variando de un caso a otro los valores de las tensiones pero no las zonasde incremento y decremento de estas.

Respecto a la evolución de la carga, se aprecia un gran incremento de la tensión entre las cargas de 100 y 200 Newton, salto que no se produce al elevar la carga a 300 Newton. Esta valoración es igualmente válida para el acero como para el titanio. En ambos casos para la carga de 100 Newton no se alcanza el limite elástico del material $(690 \mathrm{MPa}$ para el acero y 800MPa para el titanio) [5,6], mientras que para los casos de 200 y 300 Newton los valores obtenidos se encuentran a ambos lados de dicho limite, pero siempre por debajo de la tensión ultima del material (1000 MPa para el acero y 900 MPa para el titanio)[5,6].

Hasta ahora todas las características mencionadas sobre la evolución de las tensiones eran comunes a ambos materiales, pero hay que señalar que por término medio las tensiones en las placas de titanio son unos $60 \mathrm{MPa}$ superiores a las del acero.

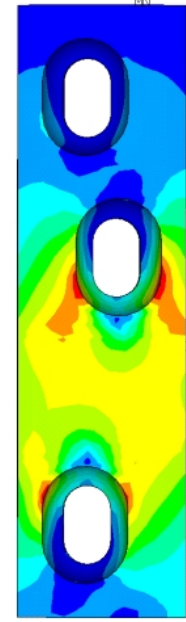

Anterior

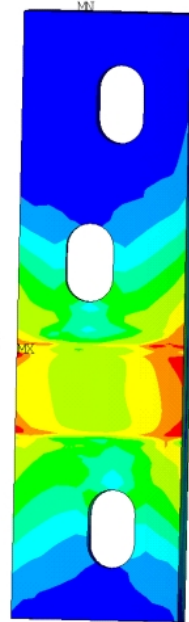

Posterior

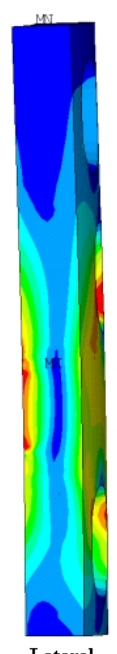

Lateral

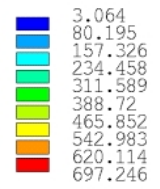

Acero: 316 LVM
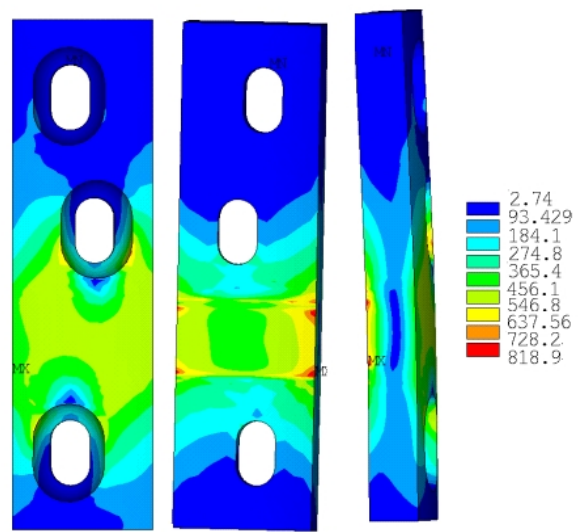

Anterior
Lateral

Titanio: Ti5Al7Nb

Figura 1. Distribución de tensiones de Von Mises en placa de acero y de titanio para $\mathrm{P}=300$, gap $=8 \mathrm{~mm}$

Comportamiento del acero y el titanio para distintos GAPs

y estados de carga

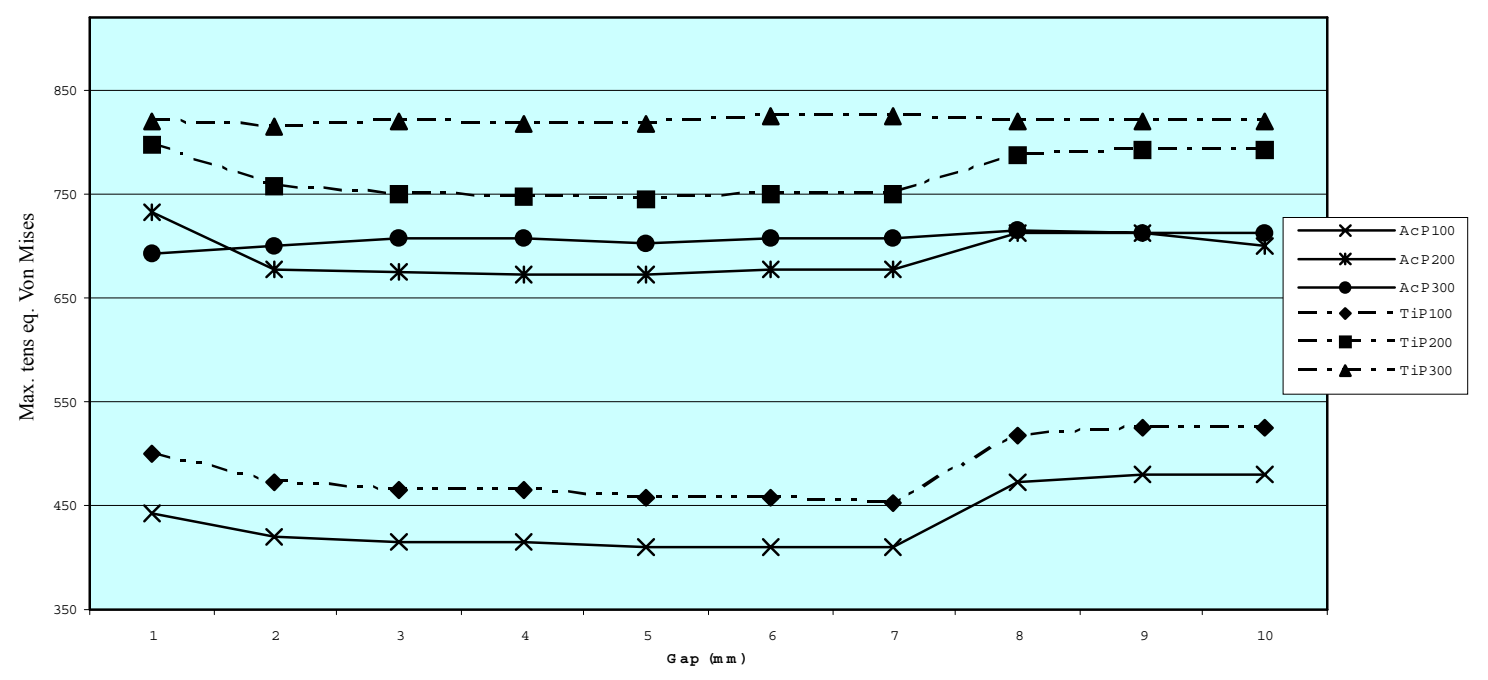

Figura 2. Evolución de las tensiones máximas en función del gap, material y carga aplicada. 


\section{Discusión y conlcusiones}

El modelo presentado supone una aproximación al estudio del comportamiento de este dispositivo al que se le han considerado suposiciones como que la placa se encuentra adherida en su totalidad a la primera cortical del hueso. En realidad, la placa se encuentra sujeta al fémur mediante tornillos, y en este sentido avanzan las actuales investigaciones: la elaboración del estudio teniendo en cuenta el contacto entre placa, tornillo y hueso.

En el presente trabajo se ha incluido la característica de deformación plástica en la pieza, tanto para el material acero como para el titanio, lo que representa un avance en la precisión del modelo. La simulación del comportamiento elasto-plástico de los materiales convierte al modelo en un problema no lineal y queda justificada porque las tensiones equivalentes obtenidas para las cargas de 200 y 300 Newton se encuentran entre estos límites.

Sobre las distribuciones de tensiones obtenidas, éstas son confirmadas por los casos reales de fallo prematuro de la pieza, ya que esta suele fallar por los bordes de los taladros mostradas en la figura 3 .

Estos primeros estudios sobre el comportamiento de las placas de osteosíntesis DCP pretenden conocer los mecanismos de fallo de estos elementos $\mathrm{y}$ averiguar que acciones podrían disminuir su tasa de fallos.

Los resultados obtenidos bajo las limitaciones comentadas, indican que la placa solo admite el apoyo parcial del miembro, con cargas inferiores a 200 Newton si consideramos como criterio de fallo su deformación plástica. Para cargas iguales o superiores a ésta, se corre el riesgo de provocar fluencia en la placa, lo que a la larga desembocará en su fallo, ya sea de acero o de titanio.

\section{Bibliografía}

1. Müller M.E.; Allgöwer M.; Manual de Osteosíntesis. Ediciones Springer-Verlag Ibérica. Tercera edición, (1993).

2. M Mae R. Diagnóstico de fracturas y principios de tratamiento. Interamericana $\mathrm{M}_{c}$ Graw-Hill, (1994).

3. M. Comín, J.L. Peris, J. Prat. Biomecáni-ca de la fractura ósea y técnicas de reparación. Instituto de biomecánica de Valencia. (1999)

4. Saura Sánchez, M.;Valverde Martinez, A. Modelización y análisis de la placa DCP en fracturas $B 2$ de fémur por el método de elementos finitos., libro actas SIB.2002.

5. Industrias Quirúrgicas del Levante. IQL. Certificado de control y conformidad. 1997.

6. Anne Hover, P.E. Technical Assessment of Materials for Fracture Fixation. http://www.depuyace.com/ dahp_app/technical_papers_list (femur)

7. M.Borgeaud, J.Cordey. Mechanical análisis of the bone to plate interface of the $L C-D C P$ and of the $P C$-FIX on human femora. Injury, Int. J. Care Injured Vol.31,(2000)

8. Renato Bombelli. Artrosis de la cadera. Clasificación y patogenia. Función de la osteotomía como terapéutica consiguiente, Salvat Editores, S.A. (1985)

9. Eiichi Genda, Norimasa Iwasaki. Normal hip joint contact pressure distribution in single-leg standing-effect of gender and anatomic parameters. Journal of Biomechanics vol. 34 (2001), 895

10. J.Cordey, M.Borgeaud. Force transfer between the plate and the bone: relative importance of the bending stiffness of the screws and the friction between plate and bone. Injury, Int. J. Care Injured Vol. 31,(2000) SC-21-28.

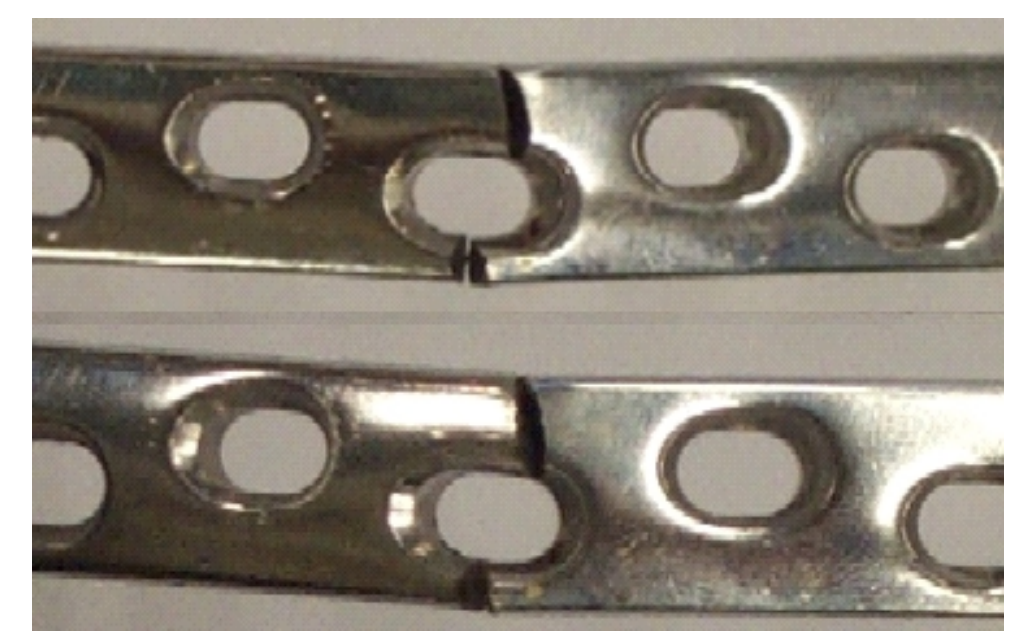

Figura 3. Placas DCP retiradas por fallo prematuro. 